\title{
A Single Institutional Phase II Randomized Trial of Whole Brain Radiation Therapy with or without Irinotecan for the Treatment of Brain Metastases from Solid Tumours
}

\author{
Soheir S. Ismail, Soheir H. Mahmoud, Dina A. Salem, Ahmed E. Essa, Diaa El-Din M. Sherif* \\ Department of Clinical Oncology and Nuclear Medicine, Faculty of Medicine, Ain-Shams University, Cairo, \\ Egypt \\ Email: *drdiaamoussa@med.asu.edu.eg, ㅎ․moussa@yahoo.com
}

Received 28 July 2015; accepted 7 September 2015; published 10 September 2015

Copyright (C) 2015 by authors and Scientific Research Publishing Inc.

This work is licensed under the Creative Commons Attribution International License (CC BY). http://creativecommons.org/licenses/by/4.0/

(c) (i) Open Access

\begin{abstract}
Background: The relatively suboptimal results of whole brain radiation therapy (WBRT) alone in eradication of brain metastases and an attempt to improve outcomes with WBRT have led to studies combining radiotherapy with chemotherapy drugs that could act as radiosensitizers with a rationale of improving local tumor control. Materials and Methods: This randomized phase II study evaluated the use of Irinotecan concomitant with 37.5 Gray (Gy) of WBRT in 2.5 Gy daily fractions $\times 5$ days each week for 3 weeks versus Whole WBRT alone in patients with brain metastasis (BM) from solid tumors. Fifty patients were randomized to receive either WBRT alone or concomitant with three irinotecan IV infusions $80 \mathrm{mg} / \mathrm{m}^{2}, 2 \mathrm{hrs}$ before RT on days 1, 8, and 15 . Results: The objective response rate (ORR) was significantly improved in patients receiving Irinotecan with radiotherapy versus radiotherapy alone $(48 \% \mathrm{vs.} 28 \% ; \mathrm{p}=0.048)$. The median time to progression of brain metastasis was significantly longer in the irinotecan and WBRT arm as compared to the WBRT arm (8 vs. 5 months; $p<0.001)$. There was no significant difference in survival between treatment arms $(p=0.361)$. Irinotecan with radiotherapy was generally well tolerated and did not interfere with the delivery of WBRT. Conclusions: Irinotecan concomitant with WBRT was well tolerated and significantly improved local control of BM compared with WBRT alone. These findings require confirmation in a phase III trial with addition of quality of life assessment.
\end{abstract}

\section{Keywords}

Brain Metastasis, Irinotecan, Whole Cranial Irradiation, Concomitant Treatment

\footnotetext{
${ }^{*}$ Corresponding author.
}

How to cite this paper: Ismail, S.S., Mahmoud, S.H., Salem, D.A., Essa, A.E. and Sherif, D.E.-D.M. (2015) A Single Institutional Phase II Randomized Trial of Whole Brain Radiation Therapy with or without Irinotecan for the Treatment of Brain Metastases from Solid Tumours. Journal of Cancer Therapy, 6, 859-870. http://dx.doi.org/10.4236/jct.2015.610094 


\section{Introduction}

Metastatic brain tumors are the most common intracranial neoplasm in adults, and although the exact incidence is unknown, it has been estimated that they can occur in up to $20 \%-40 \%$ of cancer patients during the course of their disease; an incidence 10 times greater than primary brain tumors [1]-[3].

Brain metastases are associated with poor prognosis. The median survival time of untreated patients is approximately one month, supportive care with corticosteroids extend survival to about two months, while more definitive treatments lengthen the overall median survival time to approximately four months [4] [5]. Current therapeutic approaches for BM include surgery, whole-brain radiation therapy (WBRT), stereotactic radiosurgery (SRS), chemotherapy, or a combination of these therapies [6]. Survival for patients with BM treated with WBRT typically ranges from 4 to 6 months [7] [8]. Phase III trials of the Radiation Therapy Oncology Group (RTOG) showed that treatment of brain metastasis with WBRT results in a median survival of 3 to 6 months and improve the neurologic function in most patients. No difference in median survival or 1-year survival has been seen between various dose groups, including a comparison between standard fractionation (30 Gy in 10 daily fractions) and 37.5 Gy in 15 daily fractions and accelerated hyperfractionation (1.6 Gy twice daily to 54.4 Gy) [9].

However, the relatively suboptimal results of WBRT alone in eradication of brain metastases and an attempt to improve outcomes with WBRT have led to studies combining radiotherapy with chemotherapy drugs that could act as radiosensitizers with a rationale of improving local tumor control [10]. Irinotecan is a topoisomerase I-targeted drug with bioavailability in CNS. Irinotecan also exhibits S-phase-specific cytotoxicity and radiosensitization in a variety of cancers [11]. Irinotecan is a camptothecin derivative that inhibits topoisomerase I, an essential nuclear enzyme required for relaxation of supercoiled DNA, which yields topologic changes that facilitate RNA transcription and DNA replication. Irinotecan, which crosses the blood-brain barrier, has shown activity against a range of CNS tumor xenografts in animal models. In addition, antitumor activity has been demonstrated against human glioblastoma cells with multidrug resistance [12].

Topoisomerase I (TopoI) is an essential enzyme in mammalian cells and is involved in the regulation of DNA topology during replication, recombination, and transcription. TopoI forms a phosphotyrosine bond with DNA, catalyzing a forward reaction in which DNA is cleaved to allow unwinding, and a reverse reaction in which DNA is ligated. Camptothecin and its derivatives interfere with this re-ligation step by binding to and stabilizing the enzyme/DNA complex. The resultant conversion of single strand breaks (SSBs) into irreversible doublestrand breaks (DSBs) results in cell death. TopoI inhibitors exhibit S-phase cytotoxicity and G2/M cell cycle arrest. G2/M is a relatively radiosensitive phase of the cell cycle and may in part explain the radiosensitization properties of TopoI inhibitors [13].

A Phase I/II study of irinotecan and WBRT was conducted to assess the feasibility, toxicity and efficacy of the drug for patients with brain metastases. Symptomatic improvement with radiographic response, including two complete responders, was observed in all patients with brain metastases from a variety of solid tumors [14].

On the basis of these promising results, the aim of this randomized controlled phase II study was to compare the efficacy of a combined radiochemotherapy with irinotecan and WBRT versus WBRT alone in patients with brain metastases from solid tumors in terms of response to treatment, disease progression, overall survival and toxicity of treatment.

\section{Materials and Methods}

This open label phase II randomized controlled clinical trial was carried out on 50 patients with brain metastasis from solid tumors not suitable for surgery or radiosurgery. Patients must have at least one tumor lesion that is accurately measured in at least one dimension. The Institutional Ethics Committee approved the protocol and patients were required to give informed consent before beginning the treatment.

\subsection{Eligibility Criteria}

Eligible patients for the study were 18 years and above, an Eastern Cooperative Oncology Group (ECOG) performance score of 0,1 , or, 2 who had a life expectancy $\geq 3$ months with brain metastasis from a histologically confirmed solid tumor, meeting the following criteria: Must have histologic proof of original malignancy with measurable brain metastases assessable by gadolinium-enhanced magnetic resonance imaging (MRI). The laboratory requirements were a $\mathrm{WBC} \geq 3000 / \mathrm{mm}^{3}$, an absolute neutrophil count $\geq 1500 / \mathrm{mm}^{3}$, a platelet count $\geq$ $100,000 / \mathrm{mm}^{3}$, Hemoglobin $\geq 9.0 \mathrm{~g} / \mathrm{dL}$, serum bilirubin $\leq 1.5 \mathrm{mg} / \mathrm{dL}$, AST $\leq 2.5$ times upper limit of normal or 
5 times ULN if liver metastases are present and an adequate renal functions (serum creat. $\leq 1.4$ ). Patients who had a history of previous brain radiotherapy, with germ cell tumor metastasis or prior DNA topoisomerase I drugs (e.g., irinotecan hydrochloride, topotecan hydrochloride) were excluded from the study.

\subsection{Treatment Protocol}

Fifty patients with brain metastasis from solid tumors not suitable for surgery or radiosurgery were enrolled in this study. All eligible patients were assigned in a 1:1 ratio to receive either irinotecan and whole brain radiation therapy or whole brain radiation therapy alone. All patients received a total of 37.5 Gy of WBRT in 2.5 Gy daily fractions $\times 5$ days each week for 3 weeks. In addition, patients received three irinotecan IV infusions $80 \mathrm{mg} / \mathrm{m}^{2}$, 2 hrs before RT on days 1, 8, and 15 in arm A and without addition of weekly irinotecan in arm B. WBRT used with photon energies between 1.25 and $6 \mathrm{MV}$. The primary end points of the trial were evaluation of response to treatment and evaluation of Time to CNS progression. Secondary end points were evaluation of Overall survival (OS), and assessment of toxicity.

\subsection{Evaluation of Response and Toxicity}

Pretreatment evaluation include Complete history, Clinical examination and neurologic examination, vital signs, systemic clinical examination of chest, abdomen and nervous system, performance status assessment according to the ECOG scale. Laboratory studies include Complete blood picture, liver function tests (AST, ALT, T. bilirubin. D. bilirubin), kidney function tests (s. creatinine and BUN). Radiological studies: Baseline measurement of the brain lesions with gadolinium enhancement magnetic resonance imaging (MRI). Patients visited the clinic every week and at the end of treatment for assessment of compliance, and determination of side effects. All patients underwent weekly physical and neurologic examinations and laboratory tests during treatment.

Target lesions are assessed by gadolinium-enhanced magnetic resonance imaging (Gd-MRI) 30 days after WBRT and then performed every 3 months or at time of clinical evidence of neurologic progression. Clinical and radiologic MRI evaluation of brain carried out until disease progression. Radiographic responses were assessed with gadolinium enhanced MRI according to World Health Organization (WHO) criteria;

$>$ Complete response (CR): disappearance of all known brain metastases.

$>$ Partial response (PR): $50 \%$ or greater decrease in measurable brain lesions.

$>$ Stable disease (SD): brain lesions unchanged $(<50 \%$ decrease or $<25 \%$ increase in the size of measurable lesions).

$>$ Progressive disease (PD): $>25 \%$ increase in size of some or all of brain lesions and/or the appearance of any new brain lesions.

> Objective Response Rate: include all cases of CR and PR. Time to CNS progression

(TTP) was calculated from the date of inclusion in the study to the date of documented CNS progressive disease. Overall survival (OS) was assessed from the time of registration to the date of death resulting from any cause.

Toxicity was recorded according to the Common Toxicity Criteria of the National Cancer Institute (NCI-CTC, Version 3.0) [15].

\subsection{Statistical Analysis}

Analysis of data was done by IBM computer using SPSS (statistical program for social science version 12). Intention-to-treat analyses were performed. The ORR and other categorical variables were estimated with the chisquared test or Fisher's exact test. The survival variables were estimated using the Kaplan-Meier method, and the survival rates of the treatment arms were compared using the log-rank test. In the analysis of time to progression of brain metastasis, patients who died of extracranial systemic progression were considered as free of brain metastasis if the MRI previous to the date of death did not show any brain metastasis progression. ANOVA test (analysis of variance) was used to compare more than two groups as regard quantitative variable.

\section{Results}

\subsection{Participant Characteristics}

From September/2010, to February/2012, we screened our brain metastases patients at Ain shams university 
hospitals. Of these patients, 50 met the eligibility criteria and were enrolled, with 25 patients assigned to the irinotecan and WBRT arm and 25 patients assigned to the WBRT arm, with a median follow up time of 8.5 months (range: 3 - 24 months). These patients were all included in the statistical analysis. The patient characteristics of both treatment arms are summarized in (Table 1). No statistically significant differences existed between groups at baseline.

\subsection{Response and Survival}

Tumor response is evaluated on the basis of WHO response criteria, by Gd-MRI 30 days after end of WBRT.:

Among 25 patients assessable for response in the irinotecan and WBRT arm, 12 patients (48\%) responded, including 2 (8\%) with a complete response and 10 patients (40\%) with a partial response. Of the remaining patients 10 patients (40\%) had stable disease and only 3 (12\%) patients had progressive disease. While Among 25 patients assessable for response in the WBRT arm, 7 patients (28\%), all of them had a partial response with no cases of complete response. Of the remaining patients 12 (48\%) patients had stable disease and 6 (24\%) patients had progressive disease.

These data showed that radiological complete and partial response (objective response rate) was higher (48\%) among patients in the irinotecan and WBRT arm as compared to patients in the WBRT arm (objective response rate $=28 \%$ ); while progressive disease was higher $(24 \%)$ among patients in the WBRT arm as compared to patients in the irinotecan and WBRT arm (12\%) with statistically significant difference by using chi-square test (p $=0.048$ ) (Table 2, Figure 1). Statistical analysis of factors affecting response to treatment revealed that Patients $\leq 60$ years of age had a $62.5 \%$ response rate in the combined modality arm compared to $33.33 \%$ in the WBRT arm. Patients with good ECOG performance score (score 0 and 1) had a $60 \%$ response rate in the combined modality arm compared to 33.33\% in the WBRT arm. And this indicate that the beneficial effect of irinotecan administration in improving response rate was more pronounced in patients $\leq 60$ years of age and with good performance status (ECOG score 0 and 1 ).

The median time to progression of brain metastasis was significantly longer in the irinotecan and WBRT arm as compared to the WBRT arm (8 vs. 5 months; p value < 0.001) (Figure 2).

Univariate analysis for TTP of brain metastasis in relation to different clinicopathological factors (gender, age, ECOG performance score, primary tumor type, extrecerebral metastasis and previous chemotherapy) for both study groups showed statistically significant differences for age and ECOG performance score. Age < 65 ys was associated with statistically significant higher TTP of brain metastasis as compared to age > 65 ys, and ECOG performance score 0 and 1 was associated with statistically significant higher TTP of brain metastasis as compared to ECOG performance score 2 (Table 3).

There was no significant difference in survival between treatment arms with a median overall survival of 9 months for irinotecan and WBRT arm and compared with 8 months for WBRT alone (p value =0.361). And this can be seen in (Figure 3) using the Kaplan-Meier method.

\subsection{Toxicity (Table 4)}

No statistically significant differences existed between both study arms regarding treatment related adverse events by using chi-square test. All Adverse events that were reported for patients in both treatment arms were grade 1 or 2 in severity with no reported grade 3 or 4 adverse events. The addition of irinotecan to RT was generally well tolerated, and did not interfere with the delivery of WBRT, and no neurologic acute toxicity developed in the irinotecan and WBRT arm.

As regarding hematologic adverse events neutropenia was reported in $20 \%$ of patients in the irinotecan and WBRT arm versus $8 \%$ of patients in the WBRT arm, thrombocytopenia was reported in $8 \%$ of patients in the irinotecan and WBRT arm with no thrombocytopenia reported in the WBRT arm. Anemia was reported in $24 \%$ of patients in the irinotecan and WBRT arm versus $12 \%$ of patients in the WBRT arm.

The most frequent non hematologic adverse events were alopecia reported in $68 \%$ of patients in the irinotecan and WBRT arm versus 60\% in the WBRT arm, skin hyperpigmentation reported in 60\% of patients in the irinotecan and WBRT arm versus $48 \%$ in the WBRT arm and fatigue reported in $44 \%$ of patients in the irinotecan and WBRT arm versus $40 \%$ in the WBRT arm.

Nausea was reported in $36 \%$ of patients in the irinotecan and WBRT arm versus 32\% of patients in the WBRT arm, and vomiting was reported in $24 \%$ of patients in the irinotecan and WBRT arm versus $16 \%$ of pa- 
Table 1. Comparison between both studied groups as regard general data.

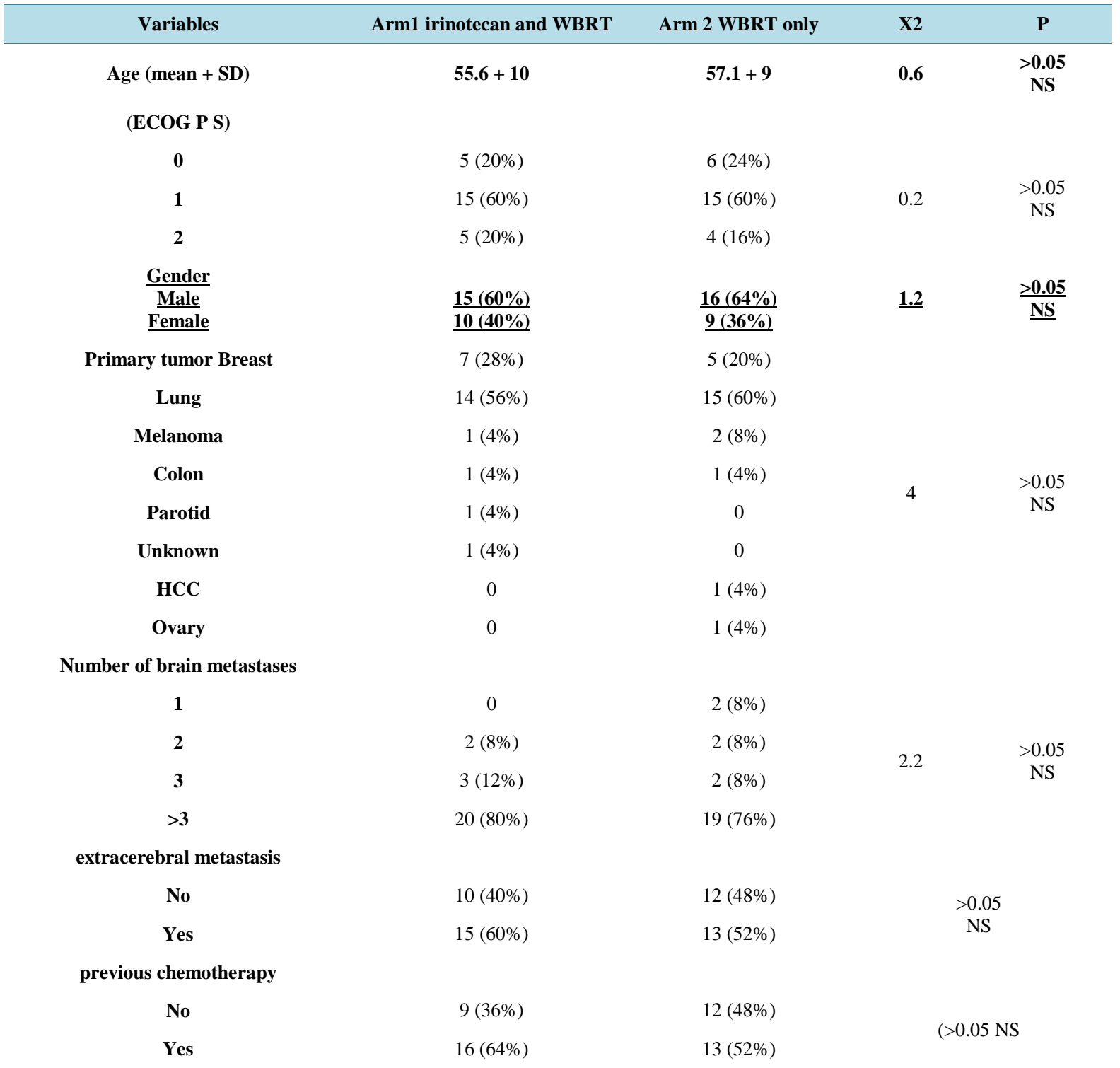

Abbreviations: ECOG, eastern cooperative oncology group, NS, not significant, PS, performance status, SD, standard deviation.

Table 2. Comparison between both studied groups as regard response to treatment 30 days after end of WBRT.

\begin{tabular}{|c|c|c|c|c|}
\hline Variables (level of response) & $\begin{array}{l}\text { Arm1 irinotecan and WBRT } \\
\qquad N=25\end{array}$ & $\begin{array}{c}\text { Arm } 2 \text { WBRT only } \\
\qquad \mathrm{N}=25\end{array}$ & OR & C.I. 95\% \\
\hline Complete response (CR) & $2(8 \%)$ & 0 & & \\
\hline Partial response (PR) & $10(40 \%)$ & $7(28 \%)$ & & \\
\hline Objective response $(\mathrm{CR}+\mathrm{PR})$ & $12(48 \%)$ & $7(28 \%)$ & 2.057 & $0.587-7.211$ \\
\hline Stable disease (SD) & $10(40 \%)$ & $12(48 \%)$ & & \\
\hline Progressive disease (PD) & $3(12 \%)$ & $6(24 \%)$ & & \\
\hline$x^{2}$ & & 6.689 & & \\
\hline p-value & & $0.048(S)$ & & \\
\hline
\end{tabular}

Abbreviations: C.I., confidence interval, OR, odds ratio, S, significant, WBRT, whole brain radiation therapy. 
Table 3. Relation between TTP of brain metastasis versus different clinicopathological variables.

\begin{tabular}{|c|c|c|c|c|}
\hline Variables & \multicolumn{2}{|c|}{$\begin{array}{c}\text { TTP } \\
\text { Mean } \pm \text { SD }\end{array}$} & $\mathbf{T}$ & $\mathbf{p}$ \\
\hline $\begin{aligned} & \text { Age } \\
< & 65 \mathrm{ys} \\
> & 65 \mathrm{ys}\end{aligned}$ & $\begin{array}{l}7.7 \\
4.5\end{array}$ & $\begin{array}{c}3 \\
2.1\end{array}$ & 2.5 & $\begin{array}{c}0.05 \\
\mathrm{~S}\end{array}$ \\
\hline $\begin{array}{c}\text { Performance } \\
0 \\
1 \\
2\end{array}$ & $\begin{array}{l}9.1 \\
7 \\
4\end{array}$ & $\begin{array}{c}2.1 \\
2 \\
1.5\end{array}$ & $\# 4.2$ & $\begin{array}{c}<0.05 \\
\mathrm{~S}\end{array}$ \\
\hline $\begin{array}{c}\text { Extrecerebral metastasis } \\
\text { No } \\
\text { Yes }\end{array}$ & $\begin{array}{l}7.7 \\
6.3\end{array}$ & $\begin{array}{c}3.5 \\
3\end{array}$ & 1.1 & $\begin{array}{c}>0.05 \\
\text { NS }\end{array}$ \\
\hline $\begin{array}{l}\text { Gender } \\
\text { Male } \\
\text { Female }\end{array}$ & $\begin{array}{l}6.5 \\
7.3\end{array}$ & $\begin{array}{c}2 \\
2.1\end{array}$ & 1.5 & $\begin{array}{c}>0.05 \\
\text { NS }\end{array}$ \\
\hline $\begin{array}{c}\text { Previous chemotherapy } \\
\text { No } \\
\text { Yes }\end{array}$ & $\begin{array}{l}6.5 \\
7.2\end{array}$ & $\begin{array}{c}1.6 \\
3\end{array}$ & 1.4 & $\begin{array}{c}>0.05 \\
\text { NS }\end{array}$ \\
\hline $\begin{array}{l}\text { Primary tumor } \\
\text { Breast } \\
\text { Lung }\end{array}$ & $\begin{array}{c}9.1 \\
7\end{array}$ & $\begin{array}{c}3.5 \\
3\end{array}$ & 1.6 & $\begin{array}{c}>0.05 \\
\text { NS }\end{array}$ \\
\hline
\end{tabular}

\#ANOVA test (F-value). Abbreviations: NS, not significant, S, significant, TTP, time to progression.

Table 4. Incidence of the main haematological and non haematological adverse events (by patients).

\begin{tabular}{|c|c|c|c|c|c|c|c|c|}
\hline & \multicolumn{4}{|c|}{ Radiochemotherapy arm } & \multicolumn{4}{|c|}{ Radiotherapy arm } \\
\hline & \multicolumn{2}{|c|}{ Grade 1} & \multicolumn{2}{|c|}{ Grade 2} & \multicolumn{2}{|c|}{ Grade 1} & \multicolumn{2}{|c|}{ Grade 2} \\
\hline Adverse Event & $\begin{array}{l}\text { NO. of } \\
\text { patients }\end{array}$ & $\begin{array}{l}\text { Percentage } \\
\text { of patients }\end{array}$ & $\begin{array}{l}\text { NO. of } \\
\text { patients }\end{array}$ & $\begin{array}{l}\text { Percentage } \\
\text { of patients }\end{array}$ & $\begin{array}{l}\text { NO. of } \\
\text { patients }\end{array}$ & $\begin{array}{l}\text { Percentage } \\
\text { of patients }\end{array}$ & $\begin{array}{l}\text { NO. of } \\
\text { patients }\end{array}$ & $\begin{array}{l}\text { Percentage } \\
\text { of patients }\end{array}$ \\
\hline \multicolumn{9}{|l|}{ Haematologic } \\
\hline Neutropenia & 5 & $20 \%$ & 0 & $0 \%$ & 2 & $8 \%$ & 0 & $0 \%$ \\
\hline Anemia & 4 & $16 \%$ & 2 & $8 \%$ & 3 & $12 \%$ & 0 & $0 \%$ \\
\hline Thrombocytopenia & 2 & $8 \%$ & 0 & $0 \%$ & 0 & $0 \%$ & 0 & $\%$ \\
\hline \multicolumn{9}{|c|}{ Non haematologic } \\
\hline Alopecia & 11 & $44 \%$ & 6 & $24 \%$ & 9 & $36 \%$ & 6 & $24 \%$ \\
\hline Nausea & 7 & $28 \%$ & 2 & $8 \%$ & 6 & $24 \%$ & 2 & $8 \%$ \\
\hline Vomiting & 5 & $20 \%$ & 1 & $4 \%$ & 4 & $16 \%$ & 0 & $0 \%$ \\
\hline Headache & 6 & $24 \%$ & 1 & $4 \%$ & 7 & $28 \%$ & 1 & $4 \%$ \\
\hline Infection & 0 & $0 \%$ & 1 & $4 \%$ & 0 & $0 \%$ & 0 & $0 \%$ \\
\hline $\begin{array}{c}\text { Skin } \\
\text { hyperpigmentation }\end{array}$ & 12 & $48 \%$ & 3 & $12 \%$ & 10 & $40 \%$ & 2 & $8 \%$ \\
\hline Fatigue & 7 & $28 \%$ & 4 & $16 \%$ & 7 & $28 \%$ & 3 & $12 \%$ \\
\hline Diarrhea & 3 & $12 \%$ & 0 & $0 \%$ & 0 & $0 \%$ & 0 & $0 \%$ \\
\hline
\end{tabular}




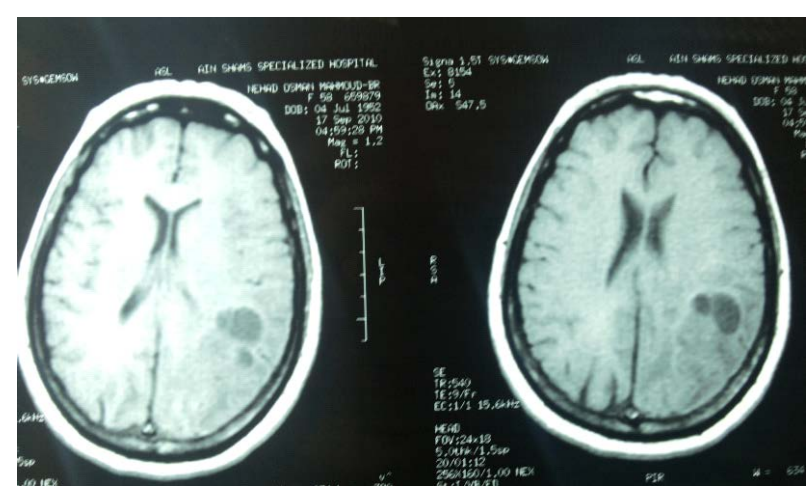

(a)

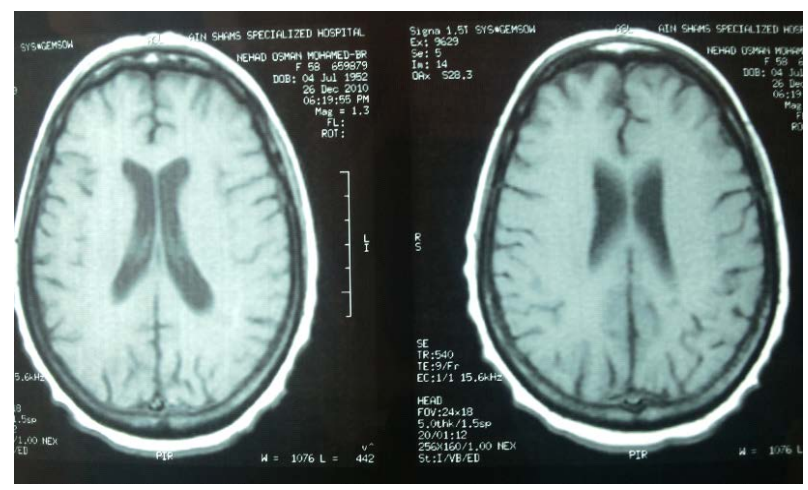

(b)

Figure 1. Axial T1-weighted MRI images after gadolinium. (a) Tow enhanced left periventricular lesions before irinotecan and whole brain radiation. (b) Complete resolution of enhancing metastasis one month after irinotecan and whole brain radiation.

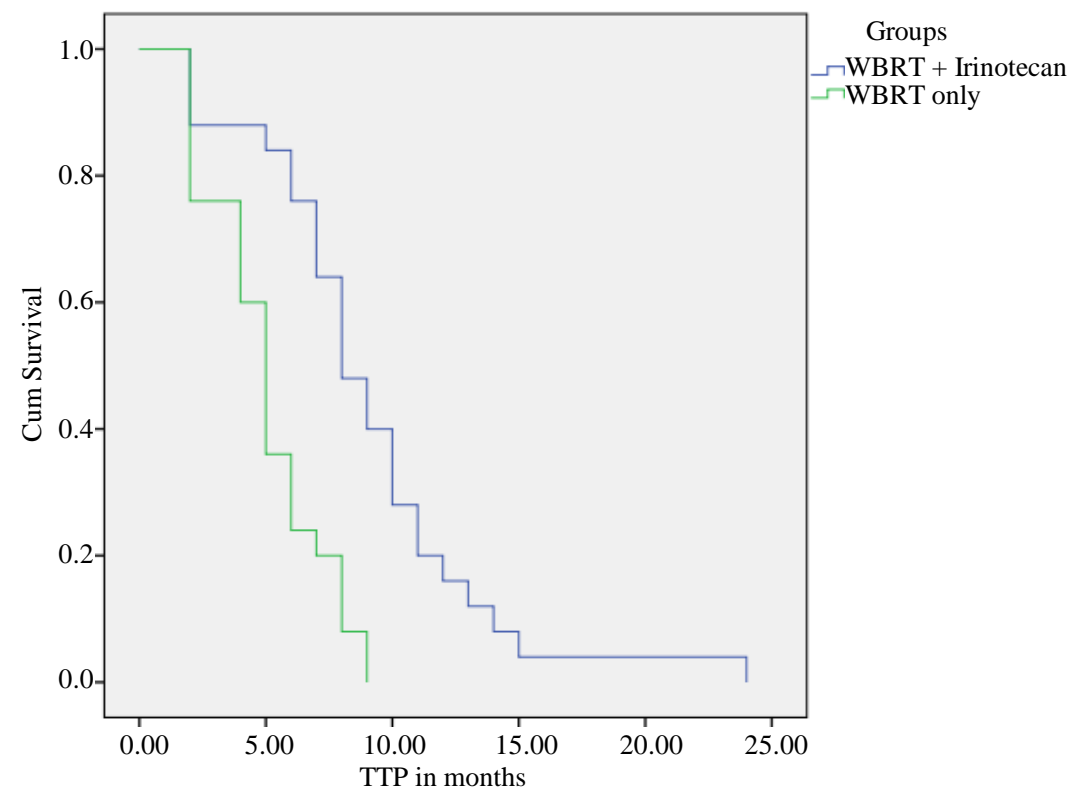

Figure 2. Kaplan Meier analysis of TTP (time to progression) of brain metastasis according to type of treatment, either WBRT (whole brain radiation therapy) plus irinotecan or WBRT only. 


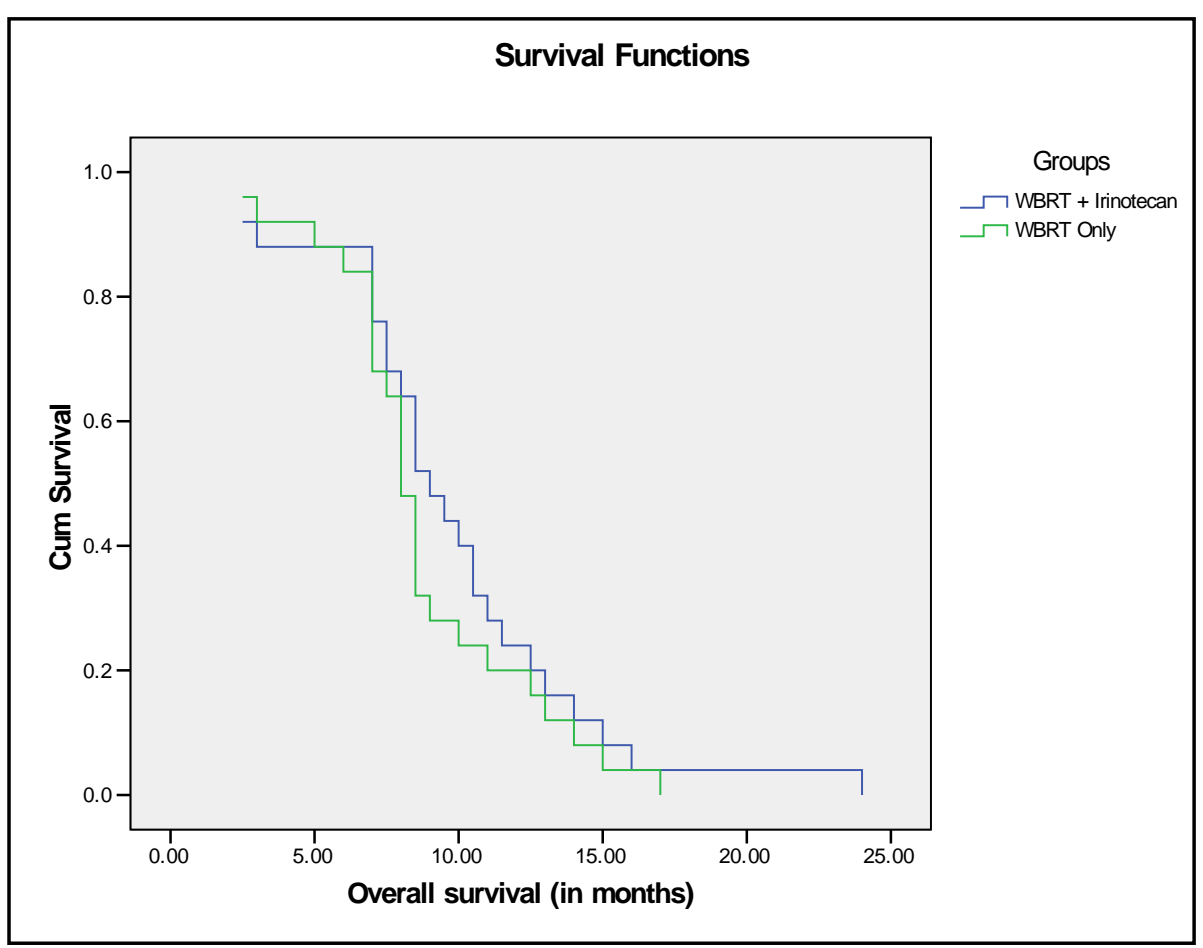

Figure 3. Kaplan-Meier Analysis of Overall Survival according to type of treatment, either WBRT (whole brain radiation therapy) plus irinotecan or WBRT only.

tients in the WBRT arm. Irinotecan induced diarrhea was reported only in $12 \%$ of patients in the irinotecan and WBRT arm, diarrhea was grade 1 and did not require irinotecan dose reduction or treatment discontinuation.

\section{Discussion}

Brain metastases are one of the most common neurologic complications of cancer. The incidence is $9 \%-17 \%$ based on various studies, although the exact incidence is thought to be higher. The incidence is increasing with the availability of improved imaging techniques which leads to early diagnosis and effective systemic treatment regimens which prolong life, thus allowing cancer to disseminate to the brain. Lung cancer, breast cancer, and melanoma are the most frequent to develop brain metastases, and account for $67 \%$ - $80 \%$ of all cancers. Most patients with brain metastases have synchronous extracerebral metastases [16].

To the best of our knowledge our study is considered to be the first randomized controlled phase II study evaluating the safety and efficacy of WBRT with irinotecan in comparison to WBRT alone for patients with brain metastases from solid tumors, with a promising statistically significant improvement in the objective response rate ( $48 \%$ vs. $28 \%$ ) and median TTP of brain metastases ( 8 vs. 5 months) in the irinotecan and WBRT arm as compared to the WBRT arm. But with insignificant improvement in the median OS (9 vs. 8 months) in the irinotecan and WBRT arm as compared to the WBRT arm. Moreover the addition of irinotecan to RT was generally well tolerated, and did not interfere with the delivery of WBRT.

Our trial comes favorably with the phase I/II trial of irinotecan and whole brain radiation therapy in patients with brain metastases from histologically documented solid tumors, conducted by Chen et al. [14] Treatment was well tolerated at the first two dose levels of irinotecan $\left(60,80 \mathrm{mg} / \mathrm{m}^{2}\right)$. No irinotecan-induced diarrhea has been observed. Symptomatic improvement with radiographic response, including two CRs, was observed in all patients with brain metastases from a variety of solid tumors and the authors concluded that concurrent irinotecan and WBRT for patients with brain metastases is well-tolerated and exhibiting promising efficacy.

Regarding ORR in our study it was higher (48\%) among patients in the irinotecan and WBRT arm as compared to patients in the WBRT arm (28\%) with statistically significant difference in contrast to the trial conducted by Stea et al., 2006 [17] in which there was no significant difference in response rate between those patients receiving only WBRT $(\mathrm{ORR}=38 \%)$ and those receiving treatment with WBRT and efaproxiral $(\mathrm{ORR}=$ 
$46 \%)$. In a recent study examined the use of WBRT plus radiosensitizer versus WBRT conducted by Rojas-Puentes et al. [18] that evaluated the efficacy and safety of radiosensitization using chloroquine (CLQ) concomitant with 30 Gray of whole-brain irradiation to treat patients with brain metastases from solid tumors. Seventythree eligible patients were randomized. Thirty-nine patients received WBRT (30 Gy in 10 fractions over 2 weeks) concomitant with $150 \mathrm{mg}$ of CLQ for 4 weeks (the CLQ arm). Thirty-four patients received the same schedule of WBRT concomitant with a placebo for 4 weeks (the control arm). The overall response rate (ORR) was $54 \%$ for the CLQ arm and $55 \%$ for the control arm ( $\mathrm{p}=0.92)$. The progression-free survival of brain metastases rates at one year were $83.9 \%$ for the CLQ arm and $55.1 \%$ for the control arm. No differences in quality of life or toxicity were found between the study arms. The median OS was 8.4 months. There was no difference in the OS between the treatment arms. In the CLQ arm, the median OS was 10.2 months, whereas the control arm median OS was 7.42 months $(\mathrm{p}=0.839)$ and the authors concluded that treatment with CLQ plus WBRT improved the control of BM (compared with the control arm) with no increase in toxicity; however, CLQ did not improve the RR or OS. These results comes comparable with our results regarding improved local control of brain metastases in the irinotecan arm with no increase in toxicity and at the same time this was not translated into prolongation of overall survival.

In the phase III multicenter randomized trial conducted b y Antonadou et al. [19] temozolamide and like irinotecan in our study achieved promising results when combined with WBRT in treatment of brain metastases from solid tumors. A significantly higher response rate was observed between the two arms, a 53.4\% response rate was seen in the combined modality arm versus $33.3 \%$ in the WBRT arm, in comparison to response rate of $48 \%$ in the combined modality arm versus $28 \%$ in the WBRT in our study. Time to progression of brain metastases as in our study was significantly improved in the combined modality arm (7.4 months) versus (5.9 months) in the WBRT arm in comparison to 8 months in the combined modality arm and 5 months in the WBRT arm in our study. Again and as in our study this significant improvement in response of brain metastases to treatment and time to cerebral progression was not translated into significant prolongation of overall survival. Median survival was 8.3 months in the temozolamide_WBRT arm and 6.3 months in the WBRT arm in comparison to median survival of 9 months in the irinotecan_WBRT arm and 8 months in the WBRT arm in our study which was insignificant in both trials.

Irinotecan and WBRT arm of our study also compares favorably with the phase II study of whole brain radiotherapy and temozolomide conducted by Addeo et al. [20] regarding brain metastases response rate and median time to CNS progression. The overall response rate was $45 \%$ and the median time to progression of brain metastases was 9 months in Addeo study in comparison to $48 \%$ overall response rate and 8 months median TTP in the irinotecan and WBRT arm of our study, with a higher median overall survival in addeo trial than in our study (13 months versus 9 months) which can be explained by better Patient characteristics in addeo trial than in irinotecan and WBRT arm of our study namely performance status, number of brain metastases, presence of extracranial metastases and primary tumor control.

In our study, age $<65$ ys was associated with statistically significant higher TTP of brain metastasis as compared to age $>65$ ys and ECOG performance score 0 and 1 was associated with statistically significant higher TTP of brain metastasis as compared to ECOG performance score 2 and this comes Compatible with the results of Addeo study that revealed in a univariate analysis for PFS a statistically significant differences for age $<65$ years, KPS $\geq 70$ and RPA class I.

Regarding the toxicity profile in our study, treatment was well tolerated. Irinotecan induced diarrhea was reported in 3 patients in the irinotecan and WBRT arm of our study, in comparison to no irinotecan-induced diarrhea has been observed in Chen et al. study. Possible explanation is that we enrolled larger number of patients in the the irinotecan and WBRT arm of our trial (25 vs. 14 patients), moreover diarrhea in our trial was grade 1 and did not require irinotecan dose reduction or treatment discontinuation.

In the study of motexafin gadolinium and whole-brain radiation therapy Mehta et al. [21] reported grade three and four adverse events: hypotension (5.8\%), asthenia (2.6\%), hyponatremia (2.1\%), leukopenia (2.1\%), hyperglycemia (1.6\%) and vomiting (1.6\%) in the WBRT and motexafin gadolinium arm. And in the In the Stea et al. trial, $28 \%$ of patients in the efaproxiral arm experienced grade 3 or 4 treatment-emergent adverse events. The most common efaproxiral severe adverse effect was hypoxemia in contrast to irinotecan and WBRT arm of our study; we did not report any grade 3 or 4 adverse events.

Our encouraging results regarding irinotecan radiosensitizing as well as cytotoxic effect when added to WBRT did not differ greatly from results of addeo et al. [22] single institution phase II trial that used a dose-in- 
tensified, protracted course of temozolomide after WBRT. The overall response rate was $48.1 \%$, the overall median survival was 8.8 months, and the median progression-free survival was 6 months in comparison to $48 \%$ response rate, 9 months overall survival and 8 months median progression-free survival in our irinotecan and WBRT arm, with an acceptable toxicity profile in both trials.

However, it is remarkable that outcomes in the irinotecan plus WBRT arm of our study were attained with a schedule that lasted for 3 weeks instead of longer treatment lengths in other clinical trials of temozolamide plus WBRT that used adjuvant temozolamide after end of radiation therapy. Consequently, our patients with extracranial metastases, with an uncontrolled primary tumor, or with both conditions were able to start or reassume chemotherapy specific for their neoplasms in a shorter time. This may have been particularly important for $36 \%$ of the patients in our study who had not received chemotherapy before the diagnosis of brain metastases. As patients with brain metastases have a short life expectancy, our treatment regimen appeared to be quite appropriate for them.

Unfortunately this local brain control was not translated into prolongation of overall survival, possible explanation may be due to a high failure rate predominantly at extra-cranial sites that erased any survival advantage that might have been the result of a better local control of brain metastases. As the majority of patients with metastatic cancer to the brain have, or soon develop, widely disseminated disease, in many cases their survival is dependent upon their overall response to cancer treatment and not on treatment for their brain metastases alone.

\section{Conclusion}

To the best of our knowledge, our study is considered to be the first randomized controlled phase II study evaluating the safety and efficacy of whole WBRT with irinotecan in comparison to WBRT alone for patients with brain metastases from solid tumors. Significantly higher objective response rates were seen with the addition of irinotecan to WBRT for the treatment of brain metastases. The beneficial effect of irinotecan administration was more pronounced in patients $\leq 60$ years of age and with good performance status (ECOG score 0 and 1 ). The addition of irinotecan to WBRT significantly delayed the progression of brain metastases with a well tolerated toxicity profile. Unfortunately this local brain control was not translated into prolongation of overall survival. These promising results of irinotecan and WBRT in treatment of brain metastases need to be confirmed in a larger phase III study with addition of quality of life and neurocognitive function assessment in the end points of the study.

\section{References}

[1] Kyritsis, A.P., Markoula, S. and Levin, V.A. (2012) A Systematic Approach to the Management of Patients with Brain Metastases of Known or Unknown Primary Site. Cancer Chemotherapy and Pharmacology, 69, 1-13. http://dx.doi.org/10.1007/s00280-011-1775-9

[2] Sheehan, J.P., Sun, M.H., Kondziolka, D., et al. (2002) Radiosurgery for Non-Small Cell Lung Carcinoma Metastatic to the Brain: Long-Term Outcomes and Prognostic Factors Influencing Patient Survival Time and Local Tumor Control. Journal of Neurosurgery, 97, 1276-1281. http://dx.doi.org/10.3171/jns.2002.97.6.1276

[3] O’Neill, B.P., Iturria, N.J., Link, M.J., et al. (2003) A Comparison of Surgical Resection and Stereotactic Radiosurgery in the Treatment of Solitary Brain Metastases. International Journal of Radiation Oncology*Biology* Physics, 55, 1169-1176. http://dx.doi.org/10.1016/S0360-3016(02)04379-1

[4] Zimm, S., Wampler, G.L., Stablein, D., et al. (2007) Intra-Cerebral Metastases in Solid Tumor Patients. Natural History and Results of Treatment. Cancer, 48, 384-394. http://dx.doi.org/10.1002/1097-0142(19810715)48:2<384::AID-CNCR2820480227>3.0.CO;2-8

[5] Xue, J., Peng, G., Yang, J.S., et al. (2013) Predictive Factors of Brain Metastasis in Patients with Breast Cancer. Medical Oncology, 30, 337. http://dx.doi.org/10.1007/s12032-012-0337-2

[6] Klos, K.J. and O’Neill, B.P. (2004) Brain Metastases. Neurologist, 10, 31-46. http://dx.doi.org/10.1097/01.nrl.0000106922.83090.71

[7] Eichler, A.F. and Loeffler, J.S. (2007) Multidisciplinary Management of Brain Metastases. Oncologist, 12, 884-898. http://dx.doi.org/10.1634/theoncologist.12-7-884

[8] Langer, C.J. and Mehta, M.P. (2005) Current Management of Brain Metastases, with a Focus on Systemic Options. Journal of Clinical Oncology, 23, 6207-6219. http://dx.doi.org/10.1200/JCO.2005.03.145

[9] Murray, K.J., Scott, C., Greenberg, H.M., et al. (2007) A Randomized Phase III Study of Accelerated Hyperfractiona- 
tion versus Standard in Patients with Unresected Brain Metastases: A Report of the Radiation Therapy Oncology Group (RTOG) 9104. International Journal of Radiation Oncology*Biology* Physics, 39, 571-574. http://dx.doi.org/10.1016/S0360-3016(97)00341-6

[10] Tsao, M.N., Lloyd, N., Wong, R.K., et al. (2012) Whole Brain Radiotherapy for the Treatment of Newly Diagnosed Multiple Brain Metastases. Cochrane Database of Systematic Reviews, 4, Article ID: CD003869. http://dx.doi.org/10.1002/14651858.cd003869.pub3

[11] Chen, A.Y., Chou, R., Shih, S.J., et al. (2004) Enhancement of Radiotherapy with DNA Topoisomerase I-Targeted Drugs. Critical Review in Oncology/Hematology, 50, 111-119. http://dx.doi.org/10.1016/j.critrevonc.2003.09.005

[12] James, J.V., Annick, D., David, A., et al. (2009) Experience with Irinotecan for the Treatment of Malignant Glioma Neuro-Oncology, 11, 80-91.

[13] Xu, Y. and Her, C. (2015) Inhibition of Topoisomerase (DNA) I (TOPI): DNA Damage Repair and Anticancer Therapy. Biomolecules, 5, 1652-1670. http://dx.doi.org/10.3390/biom5031652

[14] Chen, A.Y., Ryu, J.K. and Lau, D. (2005) A Phase I/II Trial of Irinotecan and Whole Brain Radiation Therapy in Patients with Brain Metastases. Journal of Clinical Oncology, 23, 1553.

[15] Cancer Therapy Evaluation Program, Common Terminology Criteria for adverse Events, Version 3.0, 2003. DCTD, NCI, NIH, DHHS. http://cteb.cancer.gov

[16] Nayak, L., Lee, E.Q. and Wen, P.Y. (2012) Epidemiology of Brain Metastases. Current Oncology Reports, 14, 48-54. http://dx.doi.org/10.1007/s11912-011-0203-y

[17] Stea, B., Suh, J.H., Boyd, A.P., et al. (2006) Whole-Brain Radiotherapy with or without Efaproxiral for the Treatment of Brain Metastases: Determinants of Response and Its Prognostic Value for Subsequent Survival. International Journal of Radiation Oncology*Biology*Physics, 64, 1023-1030. http://dx.doi.org/10.1016/j.ijrobp.2005.10.004

[18] Rojas-Puentes, L.L., Gonzalez-Pinedo, M., Crismatt, A., et al. (2013) Phase II Randomized, Double-Blind, PlaceboControlled Study of Whole-Brain Irradiation with Concomitant Chloroquine for Brain Metastases. Radiation Oncology, 8, 209. http://dx.doi.org/10.1186/1748-717X-8-209

[19] Antonadou, D., Coliarakis, C., Paraskevaidis, M., et al. (2002) Whole Brain Radiotherapy Alone or in Combination with Temozolomide for Brain Metastases: A Phase III Study. International Journal of Radiation Oncology*Biology* Physics, 54, 93-94. http://dx.doi.org/10.1016/S0360-3016(02)03217-0

[20] Addeo, R., Caraglia, M., Faiola, V., et al. (2007) Concomitant Treatment of Brain Metastasis with Whole Brain Radiotherapy and Temozolomide Is Active and Improves Quality of Life. BMC Cancer, 7, 18. http://dx.doi.org/10.1186/1471-2407-7-18

[21] Mehta, M.P., Rodrigus, P., Terhaard, C.H., et al. (2003) Survival and Neurologic Outcomes in a Randomized Trial of Motexafin Gadolinium and Whole-Brain Radiation Therapy in Brain Metastases. Journal of Clinical Oncology, 21, 2529-2536. http://dx.doi.org/10.1200/JCO.2003.12.122

[22] Addeo, R., Rosa, C.D., Faiola, V., et al. (2008) Phase 2 Trial of Temozolomide Using Protracted Low-Dose and Whole-Brain Radiotherapy for Nonsmall Cell Lung Cancer and Breast Cancer Patients with Brain Metastases. Cancer, 113, 2524-2531. http://dx.doi.org/10.1002/cncr.23859 


\section{List of Abbreviations}

BMs: Brain metastases,

CLQ: Chloroquine,

CR: Complete response,

ECOG: Eastern cooperative oncology group,

KPS: Karnofsky performance status,

ORR: Objective response rate,

OS: Overall survival,

PD: Progressive disease,

PR: Partial response,

RPA: Recursive partitioning analysis,

RTOG: Radiation therapy oncology group,

SD: Stable disease,

SRS: Stereotactic radiosurgery,

TopoI: Topoisomerase I.

TTP: Time to progression,

WBRT: Whole brain radiation therapy. 